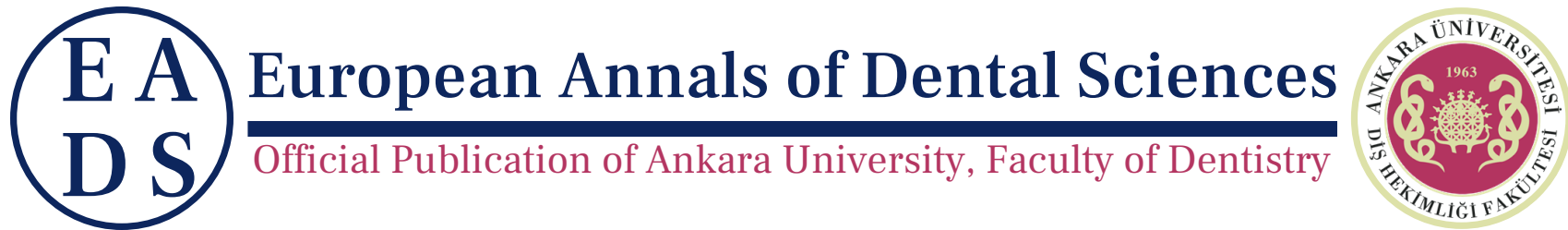

EADS, 2021, 48 (2), 69-73

\title{
Loose intra-articular body in the temporomandibular joint: case report of a successful conservative management
}

\author{
Yumi C Del Rey ${ }^{1, *}$, Hian Parize ${ }^{1}$, Christiano Oliveira-Santos ${ }^{2}$ and \\ Marina G Almeida 3
}

\begin{abstract}
${ }^{1}$ DDS, Department of Dental Materials and Prosthodontics, Ribeirão Preto Dental School, University of São Paulo (USP), Ribeirão Preto, SP, Brazil and ${ }^{2}$ DDS, MSc, PhD, Departament of Stomatology, Public Health and Forensic Dentistry, Ribeirão Preto Dental School, University of São Paulo (USP), Ribeirão Preto, SP, Brazil and ${ }^{3}$ DDS, MSc, PhD, Department of Restorative Dentistry, Ribeirão Preto Dental School, University of São Paulo (USP), Ribeirão Preto, SP, Brazil
\end{abstract}

*Corresponding Author; yumichokyu@hotmail.com

\begin{abstract}
Loose intra-articular bodies are a rare finding in the temporomandibular joint. Surgical removal of the loose bodies is often described as the only treatment option; however, it presents possible postoperative complications. The aim of this paper is to report the successful conservative management of a temporomandibular disorder associated with a single loose intra-articular body. A 62-year-old woman presented with pain in the right temporomandibular joint and pre-auricular region, limited mouth opening and open-lock episodes. Panoramic radiography and cone-beam computed tomography exams revealed the presence of a single loose body located anterior to the right condyle. Magnetic resonance images showed bilateral anterior disc displacement without reduction. A non-invasive approach consisting of an occlusal splint and low-level laser therapy associated with home-based physical therapy was performed for management of clinical signs and symptoms. The patient was followed up for 2 years and presented significant pain reduction and improvement in the range of mandibular movements. The combination of conservative therapies may be an alternative for surgical intervention to control pain and improve mandibular function in patients with temporomandibular dysfunction associated with a loose body in the temporomandibular joint.
\end{abstract}

Key words: conservative management; loose body; low-level laser therapy; temporomandibular dysfunction

\section{Introduction}

Loose intra-articular bodies (LB) are free-floating calcified masses in a joint. While commonly found in knee joint spaces, LBs affecting the temporomandibular joint (TMJ) are a rare finding.1 Common clinical symptoms are pain and swelling in the preauricular region, functional limitation, facial asymmetry, joint sounds and unilateral deviation of the mandible during mouth opening. ${ }^{1,2}$ LBs in the TMJ may originate from Inflammatory processes, e.g. osteoarthritis and rheumatoid arthritis. In these conditions, the periphery of the mandibular condyle undergo changes that lead to the formation of bony projections, the so-called osteophytes. Portions of osteophytes may subsequently become detached and lie free in the TMJ as LBs. 3,4 Trauma and benign proliferative disorders, such as synovial chondromatosis, are other possible causes of LB formation inside the TMJ. ${ }^{2,4}$

The presence of LBs is often accompanied by multiple other alterations in the TMJ. Imaging exams can reveal the existence of internal joint derangement, bone erosion, sclerosis, flattening and osteophytes; nonetheless, these findings are nonspecific. ${ }^{2,3}$ The final diagnosis can only be established based on clinical, histopathological and imaging aspects. ${ }^{1}$ Invasive procedures, such as arthroscopy and open surgery (arthrotomy), 


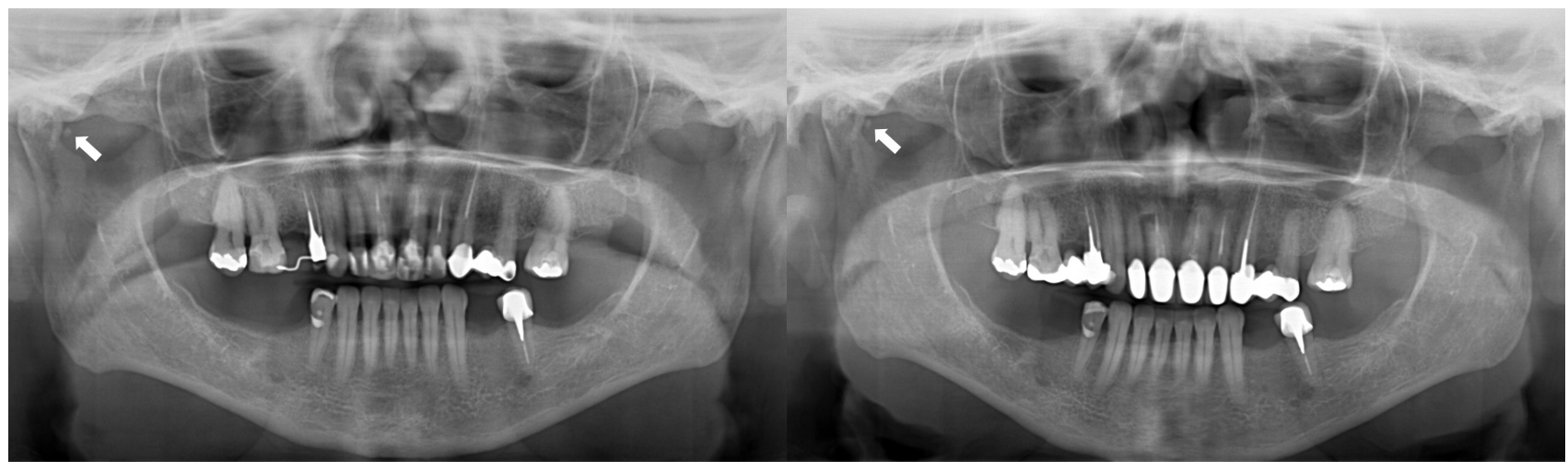

Figure 1. Panoramic radiograph shows loose solitary intra-articular body in the right temporomandibular joint space (arrow) at initial consultation (left) and 2-year follow up (right).

are the usual approaches for treatment and diagnosis, since LBs do not resolve spontaneously. ${ }^{5,6}$ These procedures consist on surgical exploration of the joint and removal of the LBs, which leads to reduction of pain and improvement of joint mobility. ${ }^{1}$ Nevertheless, surgical comorbidity should be taken in consideration, such as infection, bleeding, otologic injuries and neurovascular damages. ${ }^{7}$

The purpose of this article is to present a successful noninvasive management of signs and symptoms associated with the presence of a single LB in the TMJ. Panoramic radiography (PR), cone-beam computed tomography (CBCT) and magnetic resonance imaging (MRI) findings, the differential diagnosis and the conservative therapies performed are discussed.

\section{Case Report}

A 62-year-old female patient presented at the Temporomandibular Dysfunction Clinic of the School of Dentistry of Ribeirão Preto with pain in the right TMJ and pre-auricular region, mouth opening limitation and open-lock episodes. Clinical examination revealed limited mouth opening $(29 \mathrm{~mm})$ with corrected left deviation of mandible ( $2 \mathrm{~mm})$. A limitation in the range of mandibular protrusion, left laterality and right laterality was also detected (Table 1). Crepitus sounds were found in right and left TMJs. Pain on muscular and articular palpation was measured using a visual analog scale and was present in six of the seven areas evaluated (Table 2). Analgesics and non-steroidal anti-inflammatory drugs were previously prescribed by other dental services but did not alleviate the symptoms. Medical history was negative for osteoarthritis, rheumatoid arthritis or other joint problems. The patient had not experienced head or neck trauma.

To aid the diagnosis, a panoramic radiograph was also performed at the first consultation. The exam revealed the presence of a round radiopacity in the right condyle region (Figure 1). A CBCT scan of the right TMJ was requested and confirmed the existence of a solitary intra-articular free body $(2.9 \times 2 \times 2$ $\mathrm{mm}$ ) anterior to the right condyle, along with the presence of an osteophyte and discrete condylar cortical erosion (Figure 2). MRI of the left and right TMJs showed bilateral anterior disc displacement without reduction and the presence of osteophytes on both TMJs (Figure 3).

Based on clinical and radiological findings, osteoarthritis and synovial chondromatosis were considered as possible diagnoses. Final diagnosis could not be established due to the absence of histopathological analysis. The treatment plan comprised a combination of occlusal splint therapy, low-level laser application and home-based physical therapy. This case report was approved by the Research Ethics Committee of the

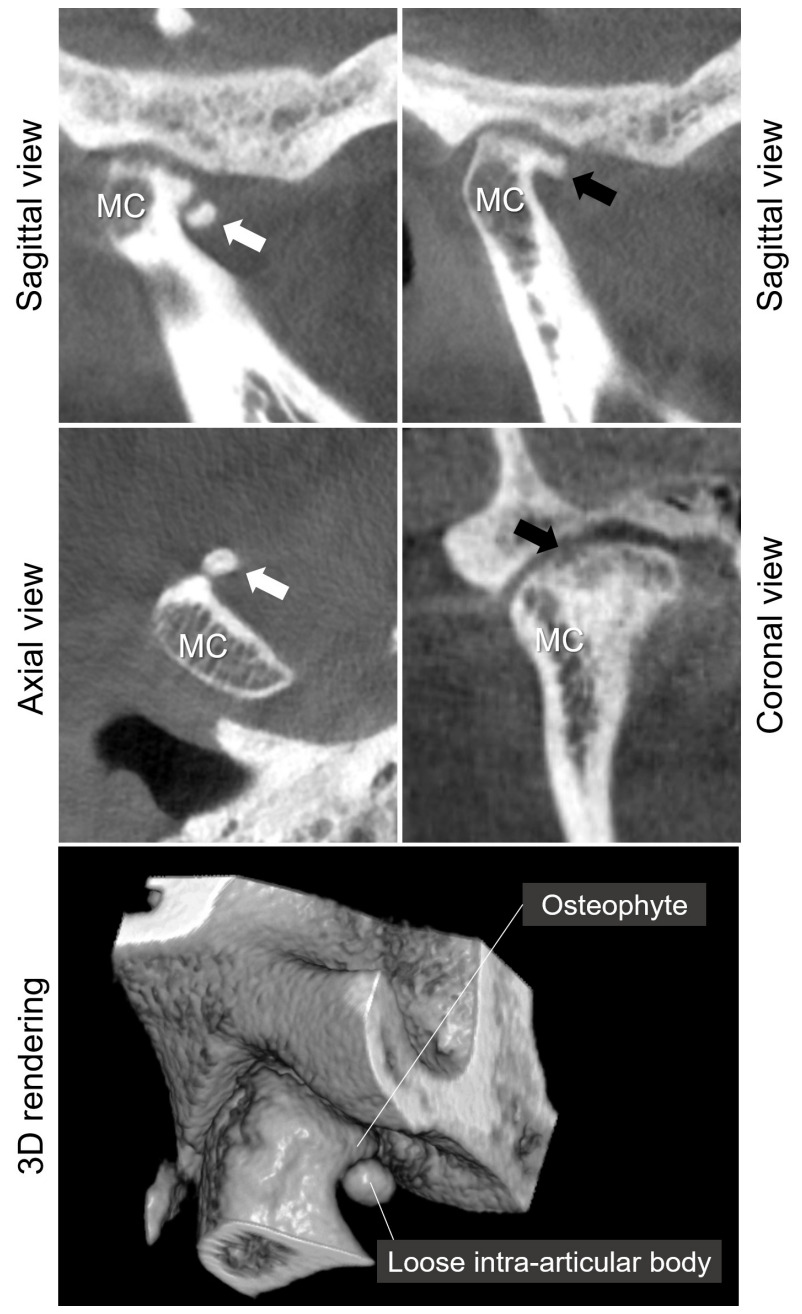

Figure 2. Cone-beam computed tomography scan of the right temporomandibular joint. The sagittal and axial views show the loose intra-articular body (yellow arrow) and osteophyte (white arrow). The coronal view shows discrete erosion of the mandibular condyle cortical (black arrow). MC: mandibular condyle.

School of Dentistry of Ribeirão Preto (University of São Paulo, Ribeirão Preto, Brazil) under the registration number CAAE 28125320.3.0000.5419. Written informed consent was obtained from the patient for the description and publication of this case report. 


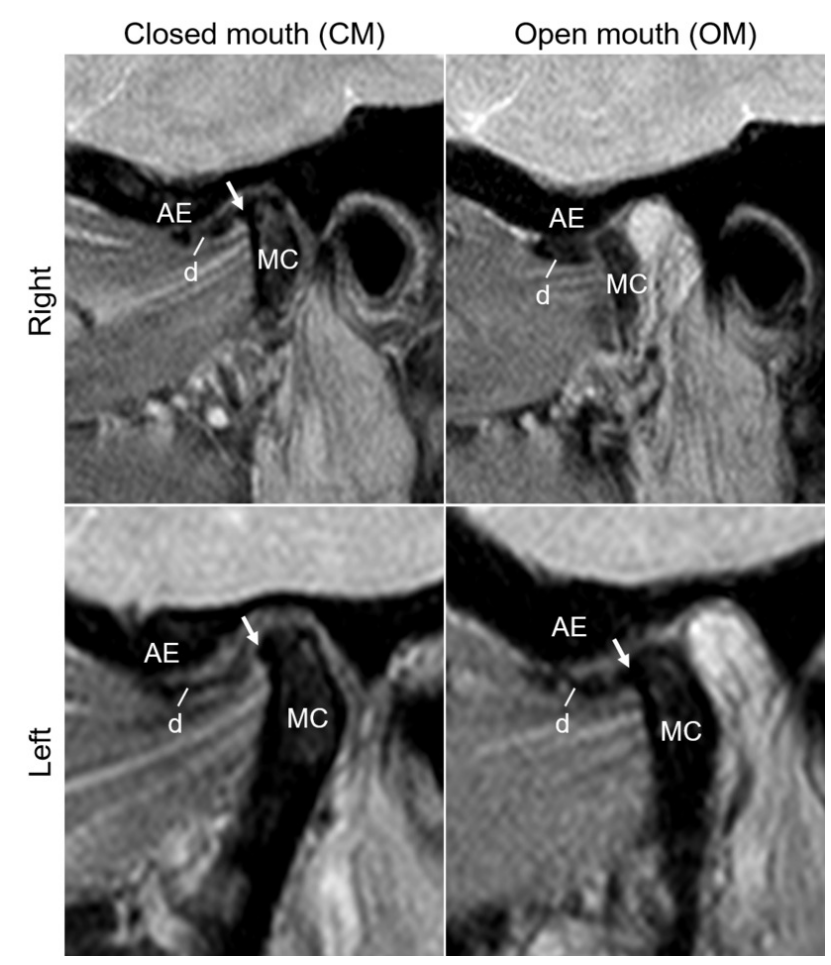

Figure 3. Sagittal proton density weighted magnetic resonance images in open and closed mouth positions of the temporomandibular joint (TMJ). On the closed mouth (CM) view of the right TMJ, the disc is located anteriorly to the mandibular condyle; the open mouth (OM) image shows there is no reduction of the disc displacement. The CM and OM views of the left TMJ also shows anterior disc displacement without reduction. The white arrows point to osteophytes. AE: articular eminence; D: articular disc; MC: mandibular condyle.

\section{Occlusal splint therapy}

An upper stabilization occlusal splint (OS) was made of transparent thermopolymerizing acrylic resin. The occlusion was adjusted until obtaining uniform and simultaneous bilateral contacts of all teeth in centric relation and lateral and anterior disocclusions by canine guidance. The patient was instructed to use the OS at night and to clean the device daily. One week after installment, the occlusal contacts were reassessed and the required adjustments were performed.

\section{Low-level laser therapy}

The low-level laser therapy (LLT) consisted of GaAlAs laser (TF Premier Plus device; MMOptics, São Carlos, Brazil) application once a week for six consecutive weeks at the following areas: masseter muscle (two points: upper and lower), anterior temporal muscle (three points: upper, middle and lower) and TMJ region (a central point and four points around the TMJ), with a distance of $1 \mathrm{~cm}$ between the application points. The laser head was positioned perpendicular to the tissues, in contact with the patient's skin. Continuous emission mode was used and the parameters were as follows: wavelength $=808 \mathrm{~nm} \pm 10$ $\mathrm{nm}$ (infrared); spot area $=0.03 \mathrm{~cm}^{2}$; time per point $=20 \mathrm{~s}$; energy density $=35 \mathrm{~J} / \mathrm{cm}^{2}$; laser optical power $=150 \mathrm{~mW}$.

\section{Home-based physical therapy and patient education}

The home-based physical therapy (PT) program comprised self-massage of masticatory muscles and application of moist heat pads on the painful areas. The self-massage was performed daily by the patient for $10 \mathrm{~min}$ with a moderate pres-
Table 1. Measurements ( $\mathrm{mm}$ ) of mandibular movements

\begin{tabular}{lccc}
\hline Mandibular movement & Baseline & $\begin{array}{c}\text { 2-year } \\
\text { follow- } \\
\text { up }\end{array}$ & Difference \\
\hline Protrusion & 9 & 11 & +2 \\
Left laterality & 8.5 & 11 & +2.5 \\
Right laterality & 7.5 & 8 & +0.5 \\
Maximum mouth opening & 29 & 34 & +5 \\
\hline
\end{tabular}

Table 2. Visual analog scale scores of pain on palpation (o to 10)

\begin{tabular}{lcccc}
\hline \multirow{2}{*}{ Palpation area } & \multicolumn{2}{c}{ Baseline } & \multicolumn{2}{c}{ 2-year follow-up } \\
\cline { 2 - 5 } & Right & Left & Right & Left \\
\hline Anterior temporal muscle & 6 & 2 & 1 & 0 \\
Posterior temporal muscle & 0 & 0 & 0 & 0 \\
Superficial masseter & 8 & 4 & 2 & 0 \\
Deep masseter & 3 & 6 & 2 & 1 \\
Trapezoid & 6 & 4 & 0 & 0 \\
Medial pterygoid & 5 & 8 & 1 & 1 \\
Pre-auricular area & 8 & 6 & 1 & 0 \\
\hline
\end{tabular}

sure using the index, middle and ring fingers in circular movements at the masseter and temporal areas, bilaterally. She was instructed to use $10 \mathrm{mg} / \mathrm{g}$ topical diclofenac potassium as an adjunct when experiencing high pain. Moist heat pads ( 40 to $50^{\circ} \mathrm{C}$ ) were used bilaterally once a day for 20 min for 7 days. The patient was oriented to avoid deleterious oral habits (e.g. biting objects, teeth clenching, provoking articular sounds) and excessive mandibular movements, to cut solid food in small pieces to avoid muscular overload and to keep the muscles relaxed and the teeth apart during rest position. The patient was also informed about the relationship between chronic pain and psychological distress. All information and instructions were written down for the patient and she was told to continue the PT even if pain-free.

\section{Treatment outcomes}

The patient was followed up weekly for the first 6 weeks for the LLLT application, after which the intervals became broader (i.e. at 1 month, 6 months, 1 year and 2 years). The OS was adjusted when necessary and home PT and patient education were reinforced at every appointment. The patient noticed a decrease in pain and headache episodes over time. After 2 years, she was asymptomatic and no open-lock episodes happened during the follow up period. Measurements of mandibular movements and reassessment of pain on palpation were performed at the 2-year follow up ((Table 1 and Table 2). A notable decrease in pain in all areas was found compared to baseline (Table 2). Moreover, the range of mandibular movements increased significantly (Table 1).

A PR was also requested to evaluate the disease progression at the 2-year follow up. No additional findings or differences in the loose intra-articular body apparent size and position were found between baseline and 2-year follow up PR (Figure 1). Since the patient was pain-free and did not present any other clinical complaint, no additional exams were performed.

\section{Discussion}

The presence of LBs in the TMJ are often related to osteoarthritis (OA), synovial chondromatosis (SC), osteochondral fractures, rheumatoid arthritis (RA) or osteochondritis dissecans (OCD).$^{2-4}$ In the present case report, osteochondral fracture 
was excluded from differential diagnosis due to absence of history of trauma. LB in OCD are believed to be the result of avascular necrosis and detachment of subchondral bone, which has also been related to previous trauma. ${ }^{4,8}$ RA can affect the TMJ, more commonly at a late phase of the disease, ${ }^{5,9}$ but the medical history for RA was negative. Based on clinical and radiological findings, OA or SC were considered as possible diagnoses.

$\mathrm{OA}$ and SC share many similar signs and symptoms e.g. pain and swelling in the preauricular region, functional limitation, facial asymmetry, joint sounds and unilateral deviation of the mandible during mouth opening. ${ }^{2,3} \mathrm{OA}$ is a degenerative joint disease characterized by cartilage degradation and abnormal remodeling of subchondral bone. Hyperplasia of the cartilage may also occur forming ossified projections (osteophytes) that may break off and lie free in the joint as LBs. ${ }^{3,10}$ TMJs affected by OA show signs of degeneration such as cortical erosion, osteophytes, flattening or sclerosis of articular surfaces and narrowing of joint space. ${ }^{11}$ The condition is believed to develop as a consequence of joint overload and/or internal derangements. ${ }^{3,11}$ It has been reported that patients presenting nonreducing disc displacements have a higher risk of developing OA. ${ }^{11}$

SC is characterized by metaplastic changes of the synovial membrane, leading to cartilaginous nodule formation. When detached, those nodules can migrate to the articular space and undergo calcification, forming LBs of varying sizes. Joint effusion, an abnormal accumulation of intra-articular fluid, is also a common finding. ${ }^{1,2}$ The mandibular condyle can present a normal morphology or degenerative changes similar to OA. ${ }^{12}$ SC etiology remains uncertain and possible associated factors are inflammation, trauma, infection and joint overload. ${ }^{2,8}$

In the present case report, final differential diagnosis could not be established due to the absence of histopathological analysis. Clinical and radiological findings are consistent with OA, yet SC could not be ruled out. In SC, metaplastic activity takes place leading to the formation of cartilaginous nodules, which grow nourished by the joint fluid, and later originate calcified bodies. Therefore, multiple LBs that slowly grow over time are typical findings of SC. ${ }^{2,12}$ There are few reports of single LBs in TMJ related to SC and, in contrast to the present case, they were large masses $(6 \times 4 \mathrm{~mm}$ and $16 \times 9 \mathrm{~mm}){ }^{6,13}$ The 2year follow-up PR also showed no apparent progression of the condition or enlargement of the LB. Moreover, evident signs of joint degenerative changes consistent with OA (e.g. osteophyte formation and cortical erosion) were found in the CBCT exam. MRI showed bilateral anterior non-reducing disc displacement, which has been associated to a higher risk of developing $\mathrm{OA},{ }^{11}$ and no joint effusion was detected.

The literature shows that TMJ OA responds positively to non-invasive treatments e.g. anti-inflammatory drugs, hyaluronic acid injections, oral splint therapy, pulsed electrical stimulation, LLLT and acupuncture. ${ }^{10}$ Nevertheless, when LBs secondary to OA or SC were present, surgery was the treatment of choice. ${ }^{1}$ LBs mechanically block joint motion, resulting in pain, mandibular deviation during mouth opening and restricted range of movements. ${ }^{2}$ In this sense, the authors hypothesized that OS therapy could be beneficial to reduce signs and symptoms, since it increases TMJ space and reduces intraarticular pressure. ${ }^{14}$ Massage therapy and heat pads were employed to lower muscle tension and increase local blood flow, therefore contributing to reduce pain. ${ }^{15,16}$ LLLT was performed due to its analgesic and anti-inflammatory effects, which can lead to pain reduction and improvement in the mandibular function. ${ }^{17}$

Only one reported case in the literature of TMD with LBs was not treated surgically. ${ }^{8}$ Soon after the diagnosis of the presence of multiple LBs, the patient presented significant reduction of signs and symptoms. The authors claimed that the spontaneous remission was probably due a change in the LBs position within the joint, and no invasive nor conservative interventions were performed. In the present case report, no apparent modification in the LB position was found at the 2-year follow up; therefore, the improvement in joint motion and pain reduction are believed to be due to the therapies performed. To the best of the authors' knowledge, this is the first report of a successful conservative management of signs and symptoms related to a $\mathrm{LB}$ in the TMJ.

\section{Conclusion}

In this case report, a conservative approach was able to control pain and improve mandibular function in a patient presenting a loose body in the TMJ. The results suggest that the combination of non-invasive therapies can successfully manage pain and functional limitations related to the presence of loose bodies in the TMJ, therefore avoiding the need for surgical intervention and patient exposure to possible postoperative complications.

\section{Acknowledgements}

The authors would like to thank Aline Fernanda Vicentin and Prof. Dr. César Bataglion for the clinical support.

\section{Author Contributions}

Del Rey YC and Parize N carried out clinical treatment; collected the data; wrote the manuscript and revised the final version of the manuscript. Oliveira-Santos $C$ analyzed the data; wrote the manuscript and revised final version of the manuscript. Almeida MG conceived the treatment plan; analyzed the data; wrote the manuscript and revised the final version of the manuscript.

\section{Conflict of Interest}

The authors declare no conflict of interest.

\section{Authors' ORCID(s)}

$\begin{array}{ll}\text { Y.C.D.R. } & 0000-0002-2833-8842 \\ \text { H.P. } & 0000-0003-0205-5892 \\ \text { C.O.S. } & 0000-0001-9936-7547 \\ \text { M.G.A. } & 0000-0001-9494-1327\end{array}$

\section{References}

1. Carls FR, von Hochstetter A, Engelke W, Sailer HF. Loose bodies in the temporomandibular joint. The advantages of arthroscopy. J Craniomaxillofac Surg. 1995;23(4):215-221. doi:10.1016/s1010-5182(05)80210-0.

2. Guarda-Nardini L, Piccotti F, Ferronato G, Manfredini D. Synovial chondromatosis of the temporomandibular joint: a case description with systematic literature review. Int J Oral Maxillofac Surg. 2010;39(8):745-755. doi:10.1016/j.ijom.2010.03.028.

3. Cömert Kiliç S, Kiliç N, Sümbüllü MA. Temporomandibular joint osteoarthritis: cone beam computed tomography findings, clinical features, and correlations. Int J Oral Maxillofac Surg. 2015;44(10):1268-1274. doi:10.1016/j.ijom.2015.06.023. 
4. Milgram JW. The classification of loose bodies in human joints. Clin Orthop Relat Res. 1977;(124):282-291.

5. Bag AK, Gaddikeri S, Singhal A, Hardin S, Tran BD, Medina JA, et al. Imaging of the temporomandibular joint: An update. World J Radiol. 2014;6(8):567-582. doi:10.4329/wjr.v6.i8.567.

6. Pastore GP, Goulart DR, Pastore PR, Prati AJ. Removal of a Solitary Synovial Chondromatosis of the Temporomandibular Joint Using Arthroscopy. J Craniofac Surg. 2016;27(4):967-969. doi:10.1097/scs.0000000000002612.

7. González-García R, Rodríguez-Campo FJ, EscorialHernández V, Muñoz-Guerra MF, Sastre-Pérez J, NavalGías L, et al. Complications of temporomandibular joint arthroscopy: a retrospective analytic study of 670 arthroscopic procedures. J Oral Maxillofac Surg. 2006;64(11):1587-1591. doi:10.1016/j.joms.2005.12.058.

8. Ercoli C, Boncan RB, Tallents RH, Macher DJ. Loose joint bodies of the temporomandibular joint: a case report. Clin Orthod Res. 1998;1(1):62-67. doi:10.1111/ocr.1998.1.1.62.

9. Sodhi A, Naik S, Pai A, Anuradha A. Rheumatoid arthritis affecting temporomandibular joint. Contemp Clin Dent. 2015;6(1):124-127. doi:10.4103/0976-237x.149308.

10. Kalladka M, Quek S, Heir G, Eliav E, Mupparapu M, Viswanath A. Temporomandibular joint osteoarthritis: diagnosis and long-term conservative management: a topic review. J Indian Prosthodont Soc. 2014;14(1):6-15. doi:10.1007/s13191-013-0321-3.

11. Campos MI, Campos PS, Cangussu MC, Guimarães RC, Line SR. Analysis of magnetic resonance imaging characteristics and pain in temporomandibular joints with and without degenerative changes of the condyle. Int J Oral Maxillofac Surg. 2008;37(6):529-534. doi:10.1016/j.ijom.2008.02.011.

12. Benslama L, Schouman $T$, Toure $S$, Chardain J, Goudot P. Synovial chondromatosis of the temporomandibular joint: Report and analysis of 12 cases. J Stomatol Oral Maxillofac Surg. 2019;120(5):476-479. doi:10.1016/j.jormas.2018.12.004.

13. Cai XY, Yang C, Chen MJ, Jiang B, Wang BL. Arthroscopically guided removal of large solitary synovial chondromatosis from the temporomandibular joint. Int J Oral Maxillofac Surg. 2010;39(12):1236-1239. doi:10.1016/j.ijom.2010.06.013.

14. Casares G, Thomas A, Carmona J, Acero J, Vila CN. Influence of oral stabilization appliances in intra-articular pressure of the temporomandibular joint. Cranio. 2014;32(3):219223. doi:10.1179/0886963413z.00000000030.

15. Gomes C, El-Hage Y, Amaral AP, Herpich CM, Politti F, Kalil-Bussadori S, et al. Effects of Massage Therapy and Occlusal Splint Usage on Quality of Life and Pain in Individuals with Sleep Bruxism: A Randomized Controlled Trial. J Jpn Phys Ther Assoc. 2015;18(1):1-6. doi:10.1298/jjpta.Vol18_001.

16. Pimentel G, Bonotto $\bar{D}$, Hilgenberg-Sydney PB. Selfcare, education, and awareness of the patient with temporomandibular disorder: a systematic review. BrJP. 2018;1:263-269.

17. Xu GZ, Jia J, Jin L, Li JH, Wang ZY, Cao DY. Low-Level Laser Therapy for Temporomandibular Disorders: A Systematic Review with Meta-Analysis. Pain Res Manag. 2018;2018:4230583. doi:10.1155/2018/4230583. 